\title{
Síntese, caracterização e estabilidade de nanopartículas de magnetita
}

\section{Synthesis, characterization, and stability of magnetite nanoparticles}

DOI: 10.46814/lajdv3n4-075

Recebimento dos originais: 01/05/2021

Aceitação para publicação: 31/06/2021

\author{
Monise Cristina Ribeiro Casanova Coltro \\ Doutora pela Universidade de São Paulo, Brasil \\ Instituição: IFG / Campus Senador Canedo \\ Endereço: GO-403, KM 7 Quinhão 12-E, Qd. 19, Lt. 1- Senador Canedo, Goiás, CEP: 75264-899 \\ E-mail: monise.coltro@ifg.edu.br \\ Warde Antonieta da Fonseca-Zang \\ Pós doutora pela Universidade de Rostock, Alemanha \\ Doutora pelo Geowissenschaft Institut (Instituto de Geologia e Mineralogia), Johannes Gutenberg \\ Universidade de Mainz, Mainz, Alemanha \\ Instituição: Programa de Mestrado Profissional - PPGTGS IFG / Campus Goiânia \\ Endereço: Rua 75, 46 Centro, Goiânia, Goiás, CEP: 74055-110 \\ E-mail: warde.zang@ifg.edu.br \\ Joachim Werner Zang \\ Pós doutor pela Universidade de Rostock, Alemanha \\ Doutor pelo Geowissenschaft Institut (Instituto de Geologia e Mineralogia), Johannes Gutenberg \\ Universidade de Mainz, Mainz, Alemanha \\ Instituição: Instituto Federal de Goiás - IFG / Campus Goiânia \\ Endereço: Rua 75, 46 Centro, Goiânia, Goiás, CEP: 74055-110 \\ E-mail: joachim.zang@ifg.edu.br \\ Danilo César Silva e Sousa \\ Químico, pelo IFG / Campus Goiânia \\ Instituição: Instituto Federal de Goiás - IFG / Campus Goiânia \\ Endereço: Rua 75, 46 Centro, Goiânia, Goiás, CEP: 74055-110 \\ E-mail: daniloze@hotmail.com
}

\section{RESUMO}

Nanopartículas de ferro são muito utilizadas em diversas áreas de pesquisa. O elemento químico ferro (Fe), sendo o quarto elemento mais abundante na crosta terrestre, e a substância mineral magnetita, com propriedade magnética, apresentam aplicações nas áreas industrial, ambiental, biomédica e de novas tecnologias. Este trabalho apresenta processo de síntese de nanopartículas partindo-se de sais precursores, bem como a caracterização dos produtos e as rotas para estabilizá-los. Os sais químicos precursores utilizados foram o cloreto férrico $\left(\mathrm{FeCl}_{3}\right)$ e o sulfato ferroso $\left(\mathrm{FeSO}_{4}\right)$ na proporção de 2:1, sob agitação por ultrassom e $\mathrm{pH}$ ácido. Para formação do precipitado de nanopartículas usou-se solução aquosa de hidróxido de sódio $(\mathrm{NaOH})$ de $\mathrm{pH} 12$. A difratometria de raio-X, mostra a presença de magnetita $\left(\mathrm{Fe}_{3} \mathrm{O}_{4}\right)$ indicada pelos picos característicos de difração em graus $2 \Theta=18^{\circ}$ (largo), $31^{\circ}$ (fino), $36^{\circ}$ (bem definido), 43, $4^{\circ}, 45^{\circ}, 53,6^{\circ}, 57,7^{\circ}, 63,3^{\circ}$. A microscopia eletrônica de transmissão mostra a morfologia dos produtos da síntese. Fatores que influenciam a estabilidade das partículas são 
agitação, o ajuste de $\mathrm{pH}$, condições de secagem. O tamanho médio das nanopartículas de magnetitas é de aproximadamente $15 \mathrm{~nm}$.

Palavras-chave: Nanoestruturas, Ultrassom, $\gamma-\mathrm{Fe}_{2} \mathrm{O}_{3}$, Óxidos de ferro, Raios-X, Microscopia eletrônica de transmissão.

\begin{abstract}
Iron nanoparticles are widely used in several research areas. The chemical element iron $(\mathrm{Fe})$, being the fourth most abundant element in the earth's crust, and the mineral substance magnetite, with magnetic properties, have applications in industrial, environmental, biomedical, and new technology areas. This work presents the process of synthesis of nanoparticles starting from precursor salts, as well as the characterization of the products and the routes to stabilize them. The precursor chemical salts were ferric chloride $\left(\mathrm{FeCl}_{3}\right)$ and ferrous sulfate $\left(\mathrm{FeSO}_{4}\right)$ in a 2:1 ratio, under ultrasound agitation and acidic $\mathrm{pH}$. For the nanoparticles growth was applied aqueous solution of sodium hydroxide $(\mathrm{NaOH})$ at $\mathrm{pH}$ 12. X-ray diffraction shows the presence of magnetite $\left(\mathrm{Fe}_{3} \mathrm{O}_{4}\right)$ indicated by characteristic diffraction peaks in degrees $2 \Theta=18^{\circ}$ (wide), $31^{\circ}$ (fine), $36^{\circ}$ (well defined), 43.4 ${ }^{\circ}, 45^{\circ}, 53.6^{\circ}, 57.7^{\circ}, 63.3^{\circ}$. Scanning electron microscopy shows the morphology of the synthesis products. Factors that influence the stability of the particles are agitation, the $\mathrm{pH}$ adjustment, and the conditions of drying. The average size of the magnetite nanoparticles is approximately $15 \mathrm{~nm}$.
\end{abstract}

Keywords: Nanostructures, Ultrasound, $\gamma-\mathrm{Fe}_{2} \mathrm{O}_{3}$, Iron oxides, X-Rays, Transmission electron microscope.

\title{
1 INTRODUÇÃO
}

Exemplos de suspensões de nanopartículas de magnetita, para as mais variadas aplicações, apresentando propriedades magnéticas em solução aquosa, foram primeiramente descritas por Elmore (1938), onde o autor descreve a magnetização em coloides ferromagnéticos. A síntese de nanopartículas de magnetita descrita pelo autor se baseia primeiramente no método de coprecipitação em solução aquosa, onde soluções dos sais de ferro nos estados de oxidação (II) e (III) são adicionados enquanto uma solução de uma base é adicionada à mistura permitindo que o pH permaneça entre 8-12.

Nanopartículas metálicas de óxidos metálicos que mais se destacam são as magnetitas ( $\gamma$ $\mathrm{Fe}_{2} \mathrm{O}_{3}$ ), pois esse óxido de ferro, estável nas condições ambientes, apresenta importância científica e tecnológica. Abaixo de um determinado valor crítico, essas nanopartículas exibem propriedades superparamagnéticas $\left(\mathrm{Fe}_{2} \mathrm{O}_{3} \mathrm{NPs}\right.$ ) e são utilizadas na biomedicina e biotecnologia como agentes de contraste em ressonância magnética, lab-on-a-chip, bioseparação magnética, como carreadores de medicamentos ou na terapia de câncer usando hipertermia magnética (SONG, 2011).

As propriedades das nanopartículas magnéticas dependem do seu tamanho e dos efeitos de suas interações, ou seja, entre a superfície e o núcleo magnético da partícula. Existe uma relação entre superfície/volume das propriedades do óxido. Outro fator, a agregação das nanopartículas magnéticas, 
tendência espontânea causada pela vasta área superficial e forte interação interparticular (TOPRAK, 2011).

Khan et al. (2011) descreveram uma síntese de nanopartículas de magnetita por coprecipitação usando como precursores os sais de ferro nos estados de oxidação (II) e ferro (III) em meio alcalino sem nenhum surfactante. As nanopartículas obtidas por estes autores apresentam tamanho entre 9 e 14 nm. O mesmo método de síntese é descrito por El Ghandoor et al. (2012), no entanto as sínteses descritas por esses autores não fazem uso do ultrassom.

Um aperfeiçoamento tem sido apresentado para a síntese de nanomagnetitas com sais precursores de cloreto férrico $\left(\mathrm{FeCl}_{3}\right)$, sulfato ferroso $\left(\mathrm{FeSO}_{4} .7 \mathrm{H}_{2} \mathrm{O}\right)$ e hidróxido de sódio $(\mathrm{NaOH})$ para controlar o valor do $\mathrm{pH} \mathrm{12,} \mathrm{sendo} \mathrm{a} \mathrm{novidade} \mathrm{uma} \mathrm{adaptação} \mathrm{de} \mathrm{agitação} \mathrm{por} \mathrm{ultrassom} \mathrm{(DA}$ FONSECA-ZANG et al., 2014).

Invernizzi et al. (2015) revisaram em seu artigo publicações científicas e grupos de pesquisa na América Latina que pesquisam sobre nanotecnologia aplicada às áreas de medicina, energia e água, extensões relevantes para atender às necessidades sociais dos países em desenvolvimento. $\mathrm{O}$ artigo mostra que os países da América Latina incorporaram essas áreas às suas agendas de nanotecnologia, mas, essas capacidades estão concentradas no Brasil e no México, e ainda que as redes regionais de cooperação são ainda vulneráveis. Eles falam que existem desafios para transpor resultados das investigações em melhorias de qualidade de vida.

Ainda, processo nanobiotecnológico para aplicação na medicina descrito por Ereno (2006) mostra nanopartículas de prata obtidas com fungo vegetal.

Baños (2017) revisou a literatura sobre magnetita como catalisador ou suporte catalítico em reações químicas de interesse científico, tecnológico e ambiental.

Nanopartículas magnéticas apresentam potencial de aplicabilidade nas mais diversas áreas devido a suas propriedades únicas, dependentes das suas dimensões, tais como alta área superficial e propriedades catalíticas. Características como toxicidade, estabilidade, recuperação e reatividade são importantes de serem consideradas, dependendo da sua aplicação ou uso final, sendo que nanopartículas podem ser usadas na indústria têxtil, na área ambiental, no tratamento de esgoto (SHAHIDI, 2019).

Nanopartículas metálicas são capazes de absorver radiação eletromagnética em determinadas faixas de comprimento de onda, que dependem das suas propriedades físicas e geométricas (BERTIN; PEROTTONI, 2020).

São ainda usadas como demulsificantes comerciais devido a sua alta área superficial e capacidade de regeneração onde $\mathrm{pH}$, hidrofobicidade e formato são alguns dos fatores chaves para demulsificantes nanoparticulados de sucesso (ADEWUNMI; KAMAL; SOLLING, 2021). 
Eskandari e Hasanzadeh (2021) descrevem uma síntese de nanopartículas de magnetita através do método de coprecipitação química, via um campo magnético alternante, com auxílio dos pulsos de ultrassom, usados separadamente e em conjunto para obtenção de nanopartículas com média de tamanho de $6 \mathrm{~nm}$. As vantagens em se utilizar nanopartículas preparadas pelo método de coprecipitação são devidas a facilidade de prepare, precursores de síntese menos e fácil aplicação (MAJIDI, 2016).

Os objetivos da pesquisa foram de desenvolver procedimentos de síntese e caracterização de nanopartículas magnéticas de magnetita.

\section{DESENVOLVIMENTO}

Os procedimentos e rotas de síntese das nanopartículas foram desenvolvidos com sais de ferro em meio alcalino, sendo observada a estabilização das nanopartículas. O método já conhecido na literatura KANG et al. (1996) foi aperfeiçoado para uma síntese com agitação de ultrassom (DA FONSECA-ZANG et al., 2014).

Foram usados sais precursores de cloreto férrico $\left(\mathrm{FeCl}_{3}\right)$ e sulfato ferroso $\left(\mathrm{FeSO}_{4} .7 \mathrm{H}_{2} \mathrm{O}\right)$, na proporção de 2:1, diluídos em 25,0 mL de água destilada e 0,85 mL de ácido clorídrico p.a. (HCl). Foi adicionado o volume de 25,0 mL de hidróxido de sódio $(\mathrm{NaOH})$ de concentração $5 \mathrm{~mol} \mathrm{~L}^{-1}$ até atingir o pH 12. Na sequência houve a formação de um precipitado escuro (nanopartículas). O ajustamento de agitação na síntese incluiu o ultrassom. Foram consideradas tanto a faixa de pH quanto a secagem, além das condições de luminosidade natural. A temperatura ambiente foi mantida.

$\mathrm{Na}$ caracterização dos produtos das sínteses foram aplicadas as técnicas de Raios-X e de Microscopia Eletrônica de Transmissão (MET):

(i) Análises de Raios-X, onde as amostras foram maceradas e dispostas em porta amostra de vidro (difração tipo método do pó). A análise foi feita em um difratômetro Shimadzu (modelo DRX6000, Japão). Foi feita varredura constante de ângulos 2-Theta, de $10^{\circ}$ a $80^{\circ}$, à taxa de 2 graus $\min ^{-1}$.

(ii) Análises de MET para caracterização morfológica dos produtos de síntese (Marca Philips, Modelo CM200, Noruega).

\subsection{DESCRIÇÃO DOS EXPERIMENTOS}

Os procedimentos aplicados nas rotas de síntese incluem a etapa de dissolução dos sais precursores, com adição num mesmo béquer contendo 25,0 $\mathrm{mL}$ de água destilada, 5,500 g de cloreto de férrico, fórmula $\mathrm{FeCl}_{3}$ (Sigma Aldrich), 2,100 g de sulfato ferroso hidratado, fórmula $\mathrm{FeSO}_{4} 7 \mathrm{H}_{2} \mathrm{O}$ (Isofar Indústria e Comércio), 0,85 mL de solução aquosa de ácido clorídrico $(\mathrm{HCl})$, concentração 12 mol L $\mathrm{L}^{-1}$. Na etapa de precipitação, foi feito o ajuste do $\mathrm{pH}$ da solução para 12 , usando-se $25,0 \mathrm{~mL}$ de 
solução de hidróxido de sódio $(\mathrm{NaOH})$, concentração $5,0 \mathrm{~mol} \mathrm{~L}^{-1}$, com o produto formado de precipitado escuro de nanopartículas de magnetita.

O ultrassom foi acionado durante o procedimento, como variante inovadora, importante na pesquisa. O experimento iniciou-se com o acionamento do aparato de ultrassom, seguido da adição de sais de ferro em solução ácida, ajuste de pH e a formação de nanopartículas de magnetita, seguida da desativação do ultrassom.

A secagem foi natural, mas foi experimentada também a secagem do produto em forno de Mufla, para verificar processos de oxidação dos compostos de óxidos de ferro (férrico e ferroso). O fluxograma da figura 1 apresenta os experimentos de síntese de nanopartículas.

Figura 1 - Fluxograma de síntese de nanopartículas de compostos de óxido ferroso-férrico $\left(\mathrm{Fe}_{3} \mathrm{O}_{4}\right)$ ou $\left(\mathrm{FeO} \cdot \mathrm{Fe}_{2} \mathrm{O}_{3}\right)$.

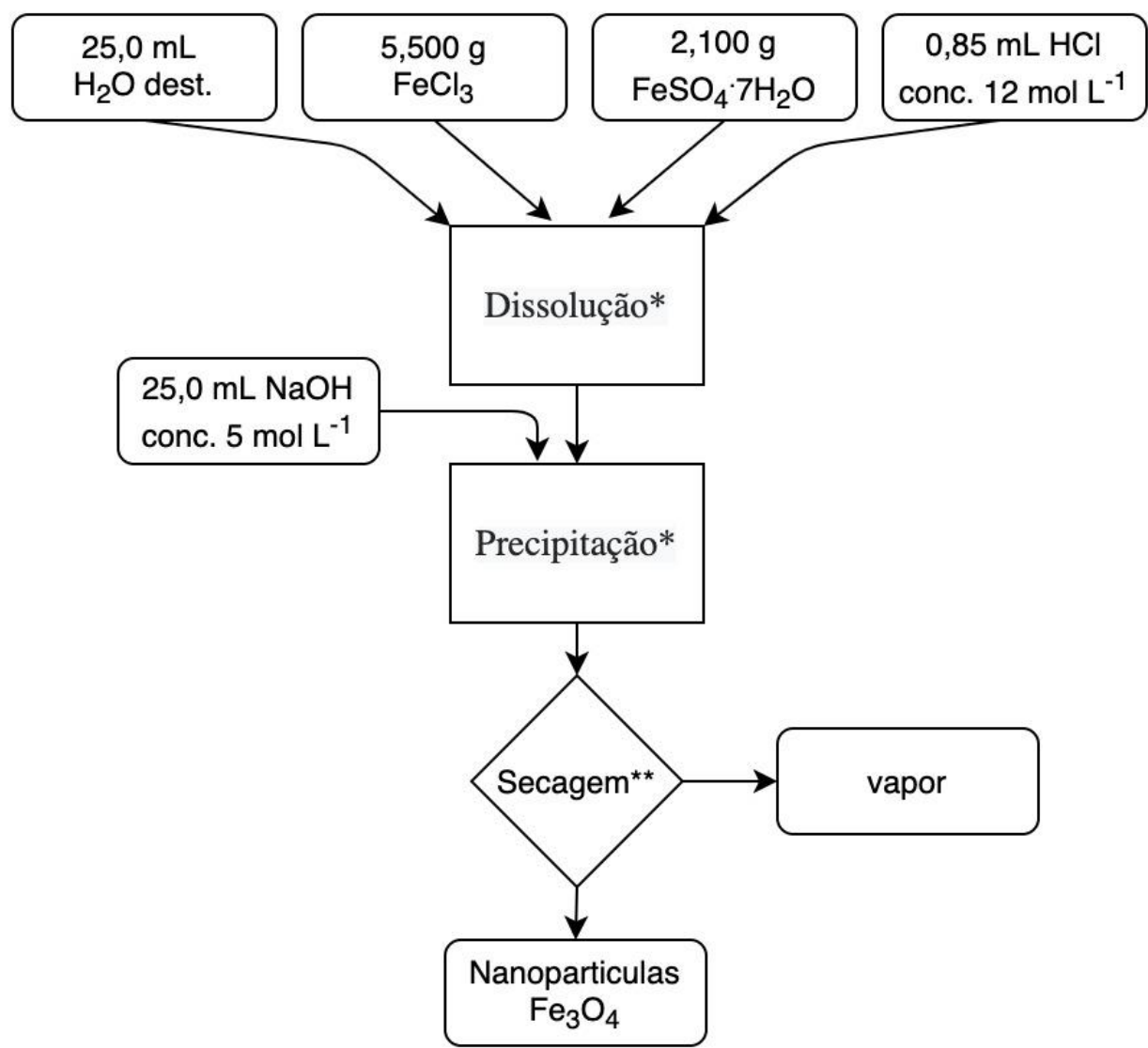

* Dissolução, seguida da precipitação no mesmo béquer, variando com acionamento de ultrassom.

** Secagem e influência no produto da síntese: secagem natural e secagem em forno de Mufla.

No fluxograma da figura 1, na rota da etapa de dissolução para a etapa de precipitação foi observada a precipitação de produtos de magnetita manométricas de melhor formação (cristalização) quando acionado o ultrassom para agitação. 


\subsection{RESULTADOS E DISCUSSÃO}

LATIN AMERICAN

LATIN AMERICAN

A síntese mostra que com acionamento do ultrassom foram produzidas partículas cristalinas dispersas. Na metodologia foi considerada a agitação por ultrassom, as concentrações das soluções e as condições de secagem. Observou-se que a secagem pode influenciar o produto da síntese de óxidos de ferro, compostos de óxido ferroso-férrico $\mathrm{Fe}_{3} \mathrm{O}_{4}$ ou $\mathrm{FeO} \cdot \mathrm{Fe}_{2} \mathrm{O}_{3}$. Na secagem no forno de Mufla, o ambiente permitiu que as partículas sintetizadas sofressem oxidação, formando óxido de ferro III (férrico), hematita $\left(\alpha-\mathrm{Fe}_{2} \mathrm{O}_{3}\right.$ ), sendo a oxidação observada (identificada) pela sua coloração vermelha característica, já a magnetita apresenta coloração preta (LEPREVOST, 1975), ver figura 2. Observouse ainda o caráter magnético com imãs, que possibilitam a observação desse efeito. Ver figura 3.

Figura 2 - Produto da síntese mostrando coloração negra avermelhada dos óxidos de ferro II e III, compostos de óxido ferroso-férrico (Fe3O4).

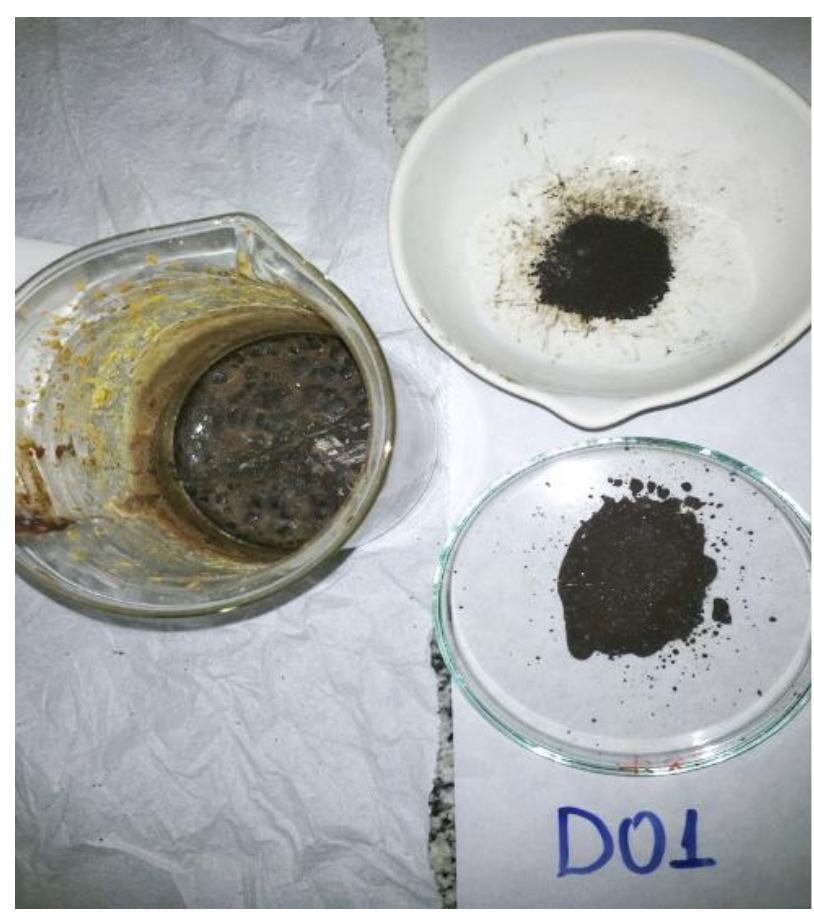


Figura 3 - Magnetismo observado com imã.

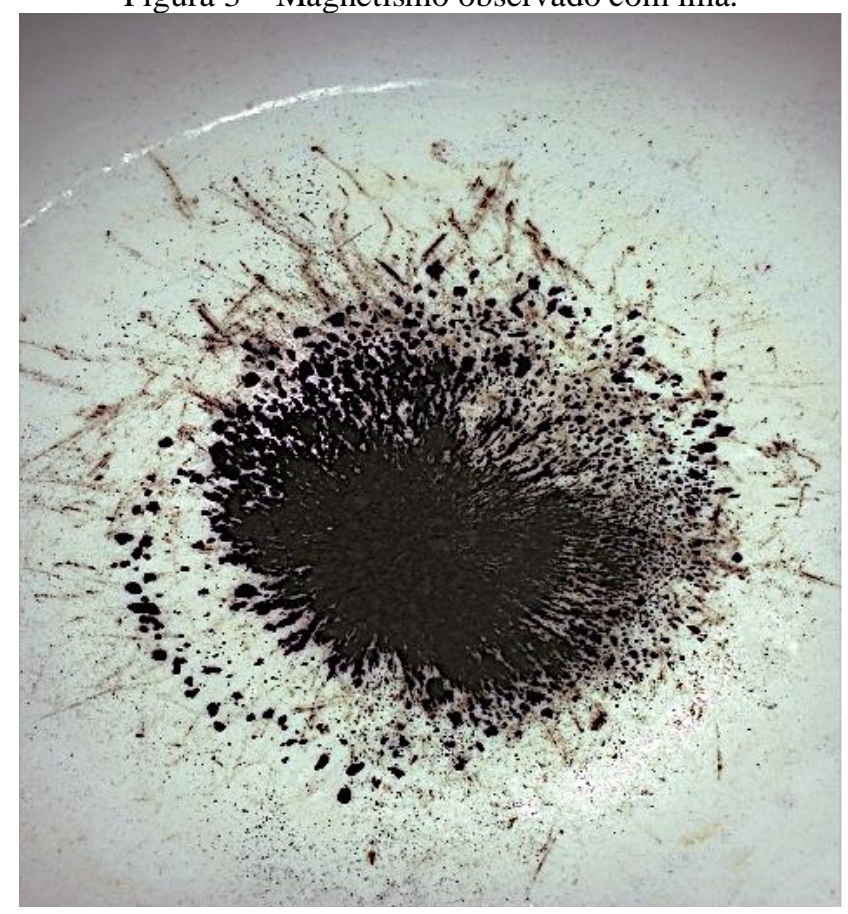

\subsection{CARACTERIZAÇÃO POR RAIOS-X:}

Feita a varredura constante de ângulos 2-Theta, de $10^{\circ}$ a $80^{\circ}$, à taxa de 2 graus $\min ^{-1}$. Os picos de difração nos difratogramas de raios-x foram comparados com os valores da ficha padrão do International Center for Diffraction Data (ICDD, 2012), JCPDS PDF database, 2012JCPDS - PDF 880866, para identificar a presença da magnetita. Os picos característicos de difração em graus $2 \Theta$ são os seguintes: $30^{\circ}$ referente as reflexões hkl (220); $35^{\circ}$ a hkl (311); 43,4 a hkl (400).

Os resultados das análises de raios-x levam a caracterizar pelo JCPDS - PDF 880866 a presença da magnetita $\left(\mathrm{Fe}_{3} \mathrm{O}_{4}\right)$, indicada pelos picos característicos de difração em graus $2 \Theta=18^{\circ}$ (largo), $31^{\circ}$ (fino), $35^{\circ}$ a hkl (311) bem definido, $43,4^{\circ}$ a hkl (400), outros $45^{\circ}, 53,6^{\circ}, 57,7^{\circ}, 63,3^{\circ}$. As análises de difração de raios-x dos produtos indicam possível presença de hematita $\left(\alpha-\mathrm{Fe}_{2} \mathrm{O}_{3}\right.$, JCPDS - PDF 871163) no pico de difração em $2 \Theta=35^{\circ}$. Ver Figura 4. 
Figura 4: Difratograma de raios-x de amostras sintetizadas com acionamento de ultrassom

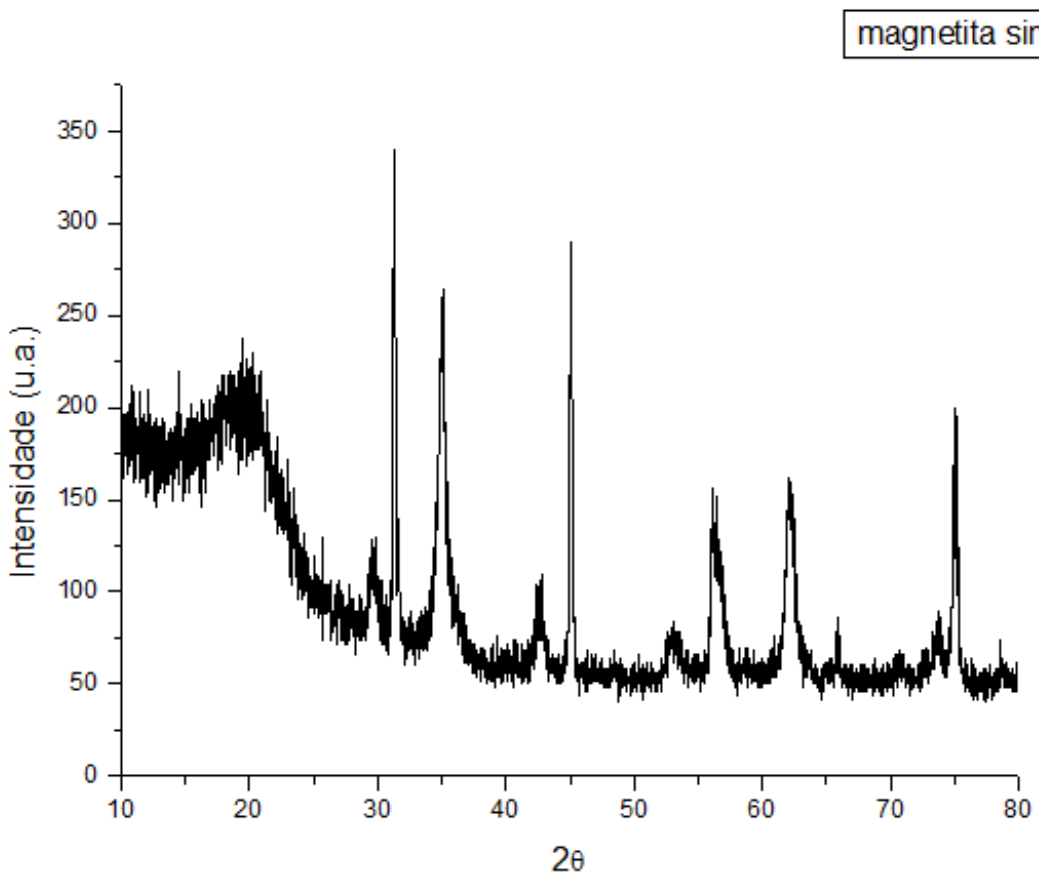

\subsection{CARACTERIZAÇÃO POR MICROSCOPIA ELETRÔNICA DE TRANSMISSÃO:}

A caracterização dos produtos pelas imagens feitas com o microscópio eletrônico de transmissão (MET) mostram nanopartículas (magnetita) produzidas nos experimentos, considerando o acionamento e não acionamento de ultrassom.

Aspectos morfológicos diferenciados e de distribuição distinta de partículas podem ser observados nas imagens de MET, tanto da figura 5, sem acionamento de ultrassom, quanto na figura 6, com acionamento da agitação por ultrassom.

Figura 5: Imagem MET de nanopartículas sem acionamento do ultrassom no experimento. Observa-se maior aglomeração.

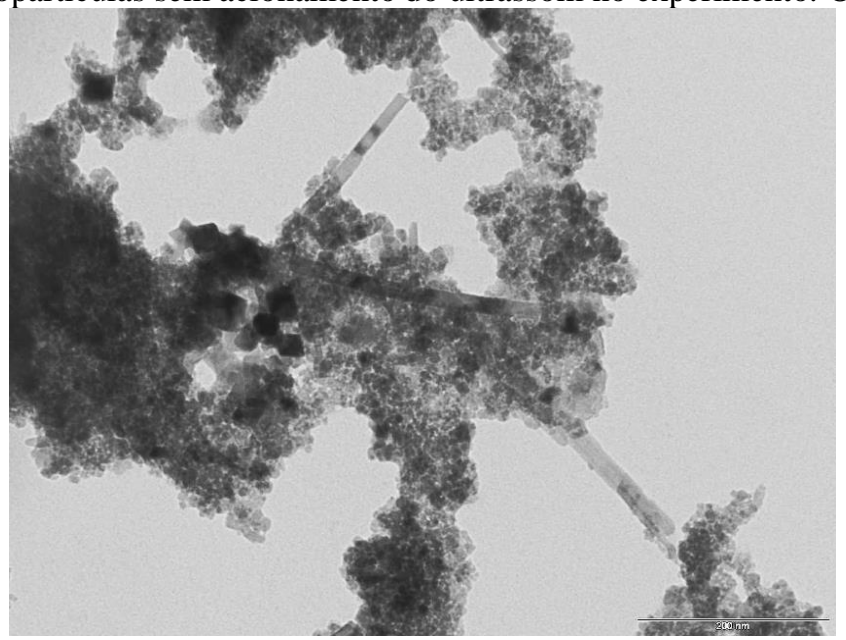


Figura 6: Imagem MET de nanopartículas com acionamento de ultrassom, dispersão

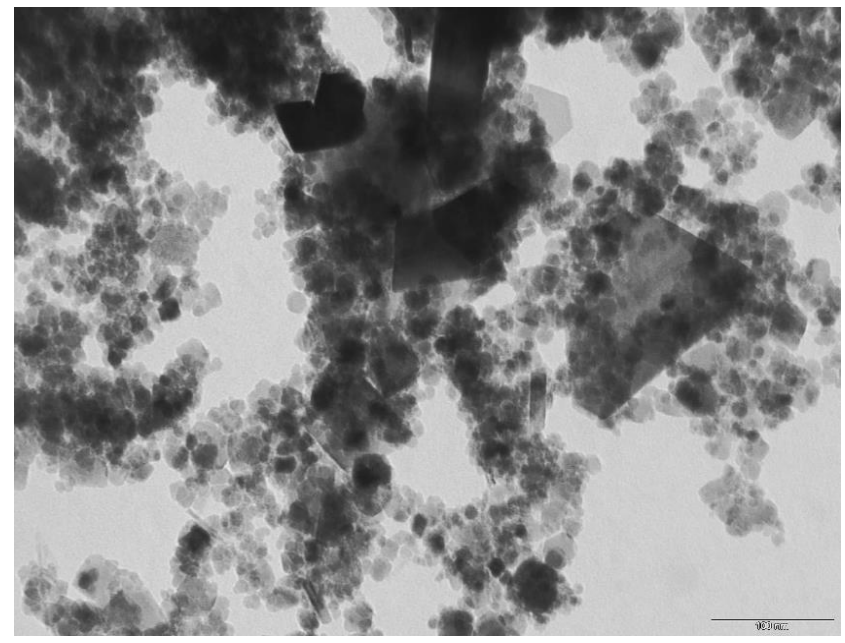

Com auxílio da técnica de MET, estima-se uma dimensão média das nanopartículas de cerca de $15 \mathrm{~nm}$. Considera-se ainda que as partículas podem apresentar dimensões variáveis, na escala manométrica, dependendo das condições da síntese. A agitação com ultrassom aperfeiçoou a síntese (Figura 7).

TOPRAK (2011) considera a agregação de nanopartículas magnéticas como espontânea, motivada pela extensa área de superfície e pela intensa interação entre as nanopartículas.

Os resultados observados para as dimensões das nanopartículas, de cerca de $15 \mathrm{~nm}$, estão de acordo com Khan et al. (2011), que sintetizaram magnetita na faixa de 9 e $14 \mathrm{~nm}$, por coprecipitação com sais de ferro (II) e (III) em meio alcalino, mas não fazendo uso de ultrassom.

Figura 7 - Imagem MET de nanopartículas de magnetita no microscópio eletrônico de transmissão (MET).

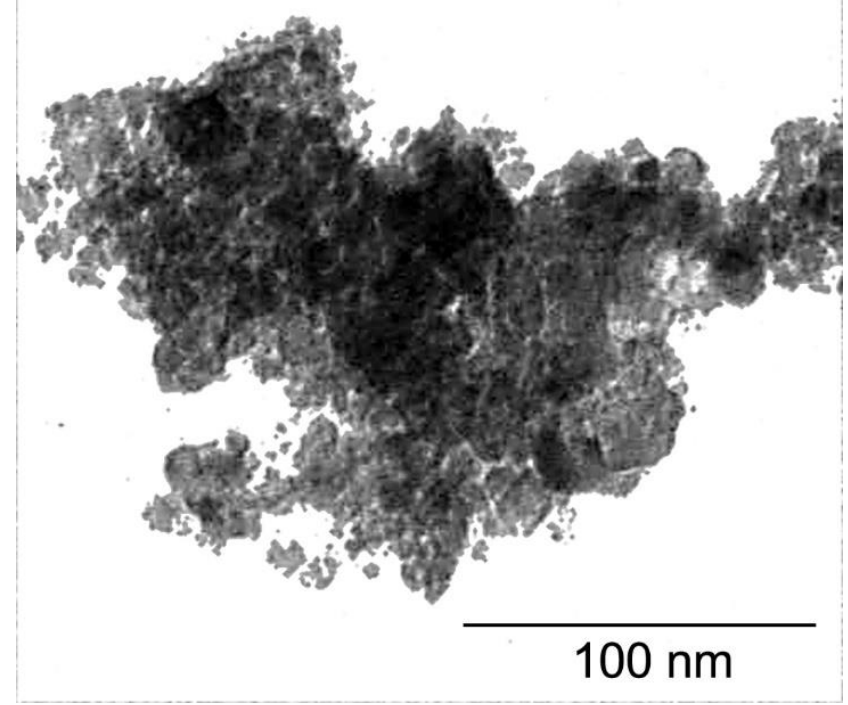




\section{CONCLUSÃO}

Os resultados mostram que a síntese de nanomagnetitas, compostos de óxido ferroso-férrico $\left(\mathrm{Fe}_{3} \mathrm{O}_{4}\right)$, a partir de sais precursores de ferro, pode ser aperfeiçoada com agitação por ultrassom. Técnicas aplicadas de raios-x e MET para caracterização dos produtos mostram que a síntese com acionamento de ultrassom leva à desaglomeração dos nanoparticulados. O ultrassom influencia o processo e seus produtos, levando a melhorar o procedimento de síntese e de dispersão dos produtos. A adaptação inovativa do ultrassom no processo de síntese de nanopartículas de magnetita contribuiu para aumentar a sua estabilidade, evitando aglutinação na cristalização. A dimensão média estimada das partículas é de $15 \mathrm{~nm}$. Difratogramas de raios-x mostram a presença predominante da magnetita $\left(\mathrm{Fe}_{3} \mathrm{O}_{4}\right)$ indicada pelos picos característicos de difração em graus $2 \Theta=18^{\circ}$ (largo), $31^{\circ}$ (fino), $36^{\circ}$ (bem definido), $43,4^{\circ}, 45^{\circ}, 53,6^{\circ}, 57,7^{\circ}, 63,3^{\circ}$.

\section{AGRADECIMENTOS}

Agradecimentos ao Instituto Federal de Educação, Ciência e Tecnologia de Goiás (IFG), Campus Goiânia, aos seus laboratórios da Área de Química pelo apoio na condução dos experimentos. Agradecimentos à Universidade Federal de Goiás (UFG): ao seu Instituto de Química, pelas análises de difratometria de raios-x, e ao seu laboratório LabMic do Instituto de Física pelas imagens feitas no microscópio eletrônico de transmissão (MET). 


\section{REFERÊNCIAS}

ADEWUNMI, A; KAMAL, M.S.; SOLLING. T. Application of magnetic nanoparticles in demulsification: A review on synthesis, performance, recyclability, and challenges. Journal of Petroleum Science and Engineering, v.196, 2021.

BAÑOS, José Gregorio Carriazo. Magnetite (Fe3O4): An inorganic structure with many applications for heterogeneous catalysis. Revista Colombiana de Química, v. 46, n.1, pp.42-59, 2017.

BATISTA DA SILVEIRA, Luciene. Estudos de propriedades magnéticas de fluídos e nanocompósitos magnéticos biocompatíveis. Brasília: UnB, set. 2006. Tese de Doutorado. Programa de Pós-graduação do Instituto de Física da Universidade de Brasília.

BERTIN, Maicon; PEROTTONI, Cláudio Antônio. Nanoplasmônica e o Espectro de Absorção de Nanopartículas Metálicas. Scientia cum industria, v.8, n.1, pp.12-24, 01 Ago. 2020.

DA FONSECA ZANG, W. A.; ZANG, J. W.; CASANOVA COLTRO, M. C. R.; BOTELHO DE OLIVEIRA, S.; SILVA E SOUSA, D.C. Processo de Síntese de nanopartículas de magnetita com uso de ultrassom. Depositante: IFG. BR 102014009136-0 A2 (2014). Depósito: 15 abr. 2014. Publicação: 24 mai. 2016.

El GHANDOOR, H.; ZIDAN, H. M.; KHALIL, M. M. H.; ISMAIL, M.I.M. Synthesis and Some Physical Properties of Magnetite (Fe3O4) Nanoparticles. International Journal of Electrochemical Science, v.7, 6 ed., p. 5734-5745, 2012.

ELMORE. W.C. The Magnetization of Ferromagnetic Colloids. Phys. Rev., v. 54, n. 309, p.1092-1095, 1938.

ERENO, D. Trama invisível: nano partículas de prata com fungo vegetal são a base de novos tecidos e fármacos. Revista Pesquisa Fapesp, ed. 122, abr. 2006. Publicação eletrônica. Disponível em: <http://lqes.iqm.unicamp.br/canal_cientifico/lqes_news/lqes_news_cit/lqes_news_2006/lqes_news_n ovidades_769_fapesp_violaceina.pdf>. Acesso em: 16 nov. 2009.

ESKANDARI, M.J., HASANZADEH, I. Size-controlled synthesis of $\mathrm{Fe}_{3} \mathrm{O}_{4}$ magnetic nanoparticles via an alternating magnetic field and ultrasonic-assisted chemical co-precipitation. Materials Science and Engineering: B, v. 266, 2021.

ICDD. International Center for Diffraction Data. JCPDS PDF database. 2012. Disponível em: <http://www.icdd.com>. Acesso em: 12 jun. 2012.

INVERNIZZI, N.; FOLADORI, G.; ROBLES-BELMONT, E.; ZAYAGO-LAU, E.; FEGUEROA, E. A.; BAGATTOLLI, C.; CARROZZA, T.; CHIANCONE, A.; URQUIJO, W. Nanotechnology for social needs: contributions from Latin American research in the areas of health, energy and water. $J$. Nanopart. Res.,17:233, 23 May 2015.

KANG, Y. S.; RISBUD, S.; RABOLT, J. F.; STROEVE, P. Synthesis and Characterization of Nanometer-Size $\mathrm{Fe}_{3} \mathrm{O}_{4}$ and $\gamma$ - $\mathrm{Fe}_{2} \mathrm{O}_{3}$ Particles. Chem. Materials, v. 8, pp. 2209-2211, 1996.

KHAN, U.S.; KHATTAK, N.S.; RAHMAN, A.; KHAN, F. Optimal Method for Preparation of Magnetite nanoparticles. Journal of the chemical society of Pakistan, v. 33, n. 5, p.628-633, 2011. 
KNECHT, M.R.; MARTINEZ, J.C.G.; CROOKS, R.M. Synthesis, Characterization, and Magnetic Properties of Dendrimer-Encapsulated Nickel Nanoparticles Containing < 150 Atoms. Chem. Mater. v.18, n.21, p.5039-5044, 2006.

LEPREVOST, A. Química Analítica dos Minerais. Rio de janeiro: Livros técnicos e científicos; Curitiba, Universidade Federal do Paraná, 1975.

MAJIDI, S.; SEHRIG, F.Z.; FARKHANI, S.M.; GOLOUJEH, M.S.; AKBARZADEH, A. Current methods for synthesis of magnetic nanoparticles. Artificial Cells, Nanomedicine and Biotechnology, v. 44, n. 2, pp. 722-734, 2016.

SCHMID, G.; PFEIL, R.; BOESE, R.; BANDERMANN, F.; MEYER, S.; CALIS, G.M.; VAN der VELDEN, J.W.A. A [Au55 P(C6H5)3 12Cl6] - A Gold Cluster of Unusual Size. Chem. Ber., v.114, p.3634-3642, 1981.

SHAHIDI, S. Magnetic nanoparticles application in the textile industry-A review. Journal of Industrial Textiles, v. 50, n. 7, pp. 970-989, May 24, 2019.

SONG, HJ.; LI, N.; SHEN, XQ. Template-free synthesis of hollow $\alpha-\mathrm{Fe}_{2} \mathrm{O}_{3}$ microspheres. Appl. Phys. $A$, v. 102, pp. 559-563, 2011.

TOPRAK, M.S. Magnetic and dielectric characterization of alginic acid- $\mathrm{Fe}_{3} \mathrm{O}_{4}$ nanocomposite. Polyedron., v.30, p.322-328, 2011. 